\title{
A WAVE FINITE ELEMENT STRATEGY TO COMPUTE THE DYNAMIC FLEXIBILITY MODES OF STRUCTURES WITH CYCLIC SYMMETRY AND ITS APPLICATION TO DOMAIN DECOMPOSITION
}

\author{
Jean-Mathieu Mencik \\ INSA Centre Val de Loire \\ Rue de la Chocolaterie, 41000 Blois, France \\ e-mail: jean-mathieu.mencik@insa-cvl.fr
}

Keywords: Wave finite element method, Periodic structures, Cyclic symmetry, Dynamic flexibility modes, Domain decomposition.

\begin{abstract}
A wave-based numerical approach is proposed for modeling periodic structures with cyclic symmetry. Wave modes which travel around the circumferential direction of those structures are calculated with the wave finite element method. Emphasis is placed on building the matrices of dynamic flexibility modes of the periodic structures by considering unit forces which are successively applied to the degrees of freedom of their boundaries. As it turns out, the matrices of dynamic flexibility modes may be quickly computed, leading the way to efficient domain decomposition techniques to analyze assemblies made up of several periodic structures.
\end{abstract}




\section{INTRODUCTION}

Periodic structures with cyclic symmetry are mechanical components which are frequently encountered in the aeronautic industry in the design of turbines. The finite element (FE) method [1] and the theory of cyclic symmetry [2] are well known approaches for modeling those systems. However, both suffer from limitations, which concern: (i) the numerical cost involved when assembling and inverting large-sized sparse matrices in an FE model; (ii) the lack of accuracy due to the fact that the coupling effects between the harmonic modes of two connected periodic structures are not taken into account in the theory of cyclic symmetry. The present paper aims at proposing an alternative approach which circumvents those issues.

In this paper, a wave-based numerical approach is proposed for computing the matrices of dynamic flexibility modes of periodic structures with cyclic symmetry. The strategy consists in analyzing the dynamic response of a structure when unit forces are successively applied to the boundary degrees of freedom (DOFs), e.g., those located on the inner circumferential boundary. Such an analysis may be quickly achieved using the wave finite element (WFE) method by considering the wave modes which travel around the circumferential direction of the structure. The matrices of dynamic flexibility modes of several periodic structures provide an efficient means to undertake domain decomposition analysis.

The WFE method is nothing else but a transfer matrix method which, once combined with the Bloch theorem, provides wave modes which propagate along periodic structures, i.e., structures composed of identical substructures along a straight or circumferential direction (see [3, 4, 5, 6, 7. 8, 9, 10, 11]). The WFE method has been further used to describe the dynamic response of periodic structures. The strategy consists in expanding the vectors of displacements and forces of a structure on a vector basis of wave modes, and using periodicity assumption to derive small matrix systems which can be solved efficiently (see [12, 13, 14, 15, 16, 17]).

One main limitation of the WFE method, however, is that the excitations are supposed to be confined to the structure ends. The present work aims at tackling this problem within the specific scope of periodic structures with cyclic symmetry whose circumferential boundaries are subject to excitations. This leads the way to the analysis of assemblies made up of several periodic structures which may be thus connected around their circumferential boundaries.

The rest of the paper is organized as follows. The FE modeling of a periodic structure with cyclic symmetry is presented in Section 2.1. The WFE method is presented in Sections 2.2 and 2.3 with a view to computing the wave modes and assessing the forced response of the structure. The derivation of the matrix of dynamic flexibility modes is carried out in Section 2.4. The domain decomposition technique which uses the concept of matrices of dynamic flexibility modes of several periodic structures is proposed in Section 2.5, Numerical experiments are finally brought in Section 3, which concern the study of one single periodic structure as well as the study of an assembly made up of two periodic structures with different periodicity patterns. Also, comparisons with the FE method and the theory of cyclic symmetry are carried out.

\section{THEORY}

\subsection{Problem description}

The FE mesh of a periodic structure with cyclic symmetry, composed of identical substructures, is shown in Figure 1, Denote as $\mathbf{D}=-\omega^{2} \mathbf{M}+(1+i \eta) \mathbf{K}$ the dynamic stiffness matrix of a substructure, with $\omega$ the angular frequency, $\mathbf{M}$ the mass matrix, $\mathbf{K}$ the stiffness matrix, $\eta$ the loss factor and $i$ the unit imaginary number. As it turns out, the dynamic equilibrium matrix 
equation of the substructure is given by:

$$
\left[\begin{array}{ll}
\mathbf{D}_{\Gamma \Gamma} & \mathbf{D}_{\Gamma \mathrm{I}} \\
\mathbf{D}_{\mathrm{I} \Gamma} & \mathbf{D}_{\mathrm{II}}
\end{array}\right]\left[\begin{array}{l}
\mathbf{q}_{\Gamma} \\
\mathbf{q}_{\mathrm{I}}
\end{array}\right]=\left[\begin{array}{l}
\mathbf{F}_{\Gamma} \\
\mathbf{F}_{\mathrm{I}}
\end{array}\right]
$$

where $\mathbf{q}$ and $\mathbf{F}$ are vectors of nodal displacements and nodal forces, respectively, while subscripts $\Gamma$ and I refer to interface DOFs and internal DOFs, respectively (see Figure1).
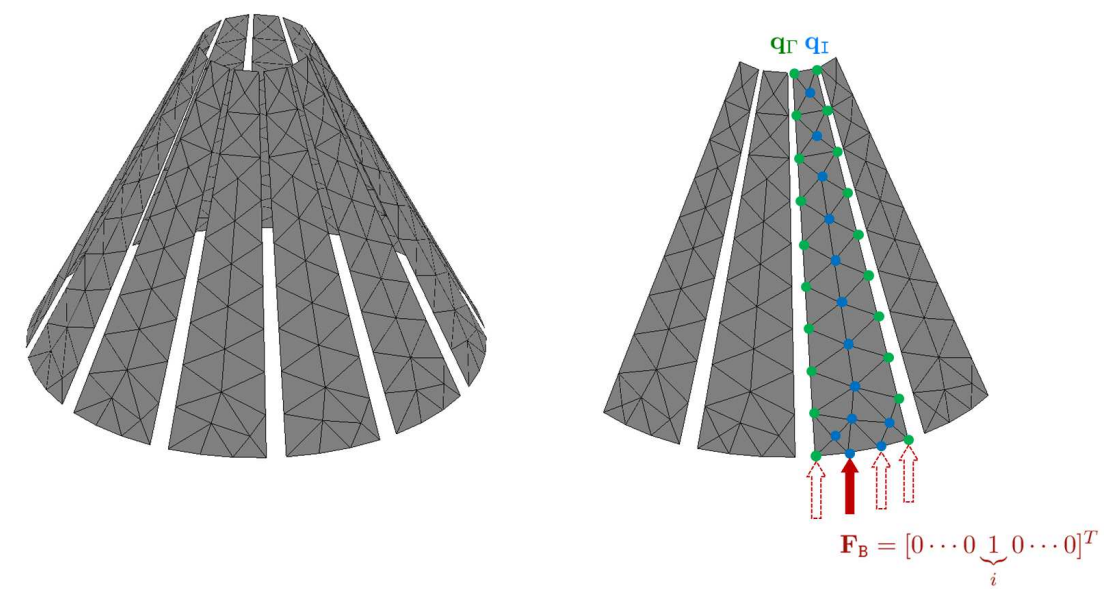

Figure 1: Periodic structure with one substructure excited by a unit force applied to one boundary DOF (the boundary of the substructure is here considered as being the bottom side).

Consider a substructure which is excited by a unit force on one of its boundary DOFs - say, DOF $i$ - as shown in Figure 1. The boundary DOFs of the substructure are to be understood like those where coupling with other structural components may occur. Here, the vector of boundary forces of the substructure is defined as

$$
\mathbf{F}_{\mathrm{B}}=[0 \cdots 0 \underbrace{1}_{i} 0 \cdots 0]^{T} \text {. }
$$

Notice that the boundary DOFs may represent subparts of the interface and internal DOFs, as shown in Figure 1. Thus, by considering the vector of boundary forces $\mathbf{F}_{\mathrm{B}}$, Eq. (1) may be rewritten as follows:

$$
\left[\begin{array}{ll}
\mathbf{D}_{\Gamma \Gamma} & \mathbf{D}_{\Gamma \mathrm{II}} \\
\mathbf{D}_{\mathrm{I \Gamma}} & \mathbf{D}_{\mathrm{II}}
\end{array}\right]\left[\begin{array}{l}
\mathbf{q}_{\Gamma} \\
\mathbf{q}_{\mathrm{I}}
\end{array}\right]=\left[\begin{array}{c}
\mathbf{F}_{\Gamma}+\mathcal{L}_{\Gamma \mathrm{B}} \mathbf{F}_{\mathrm{B}} \\
\mathcal{L}_{\mathrm{IB}} \mathbf{F}_{\mathrm{B}}
\end{array}\right]
$$

where $\mathcal{L}_{\Gamma \mathrm{B}}$ and $\mathcal{L}_{\mathrm{IB}}$ are Boolean localization matrices, and $\mathrm{F}_{\Gamma}$ is the vector of coupling forces between substructures. By condensing the dynamic equilibrium equation (3) of the excited substructure w.r.t. the interface DOFs, this yields:

$$
\mathbf{D}^{*} \mathbf{q}_{\Gamma}=\mathbf{F}_{\Gamma}+\left(\mathcal{L}_{\Gamma \mathrm{B}}-\mathbf{D}_{\Gamma \mathrm{I}} \mathbf{D}_{\mathrm{II}}^{-1} \mathcal{L}_{\mathrm{IB}}\right) \mathbf{F}_{\mathrm{B}}
$$

where $\mathbf{D}^{*}=\mathbf{D}_{Г Г}-\mathbf{D}_{\Gamma \mathrm{I}} \mathbf{D}_{\mathrm{II}}^{-1} \mathbf{D}_{\mathrm{I} \Gamma}$ is the condensed dynamic stiffness matrix of the substructure. Notice that, for a non-excited substructure $\left(F_{B}=0\right)$, Eq. (4) gives:

$$
\mathbf{D}^{*} \mathbf{q}_{\Gamma}=\mathbf{F}_{\Gamma} .
$$

Eq. (5) is the starting point of the WFE method which will be developed hereafter. 


\subsection{WFE method}

Consider a periodic structure with a rotational periodicity of $2 \Delta \theta$ as shown in Figure 2 , and denote as $(k)(k=1,2, \ldots)$ the coupling interfaces between the substructures. Also, assume that the substructures are free of excitation sources other than coupling forces between substructures.

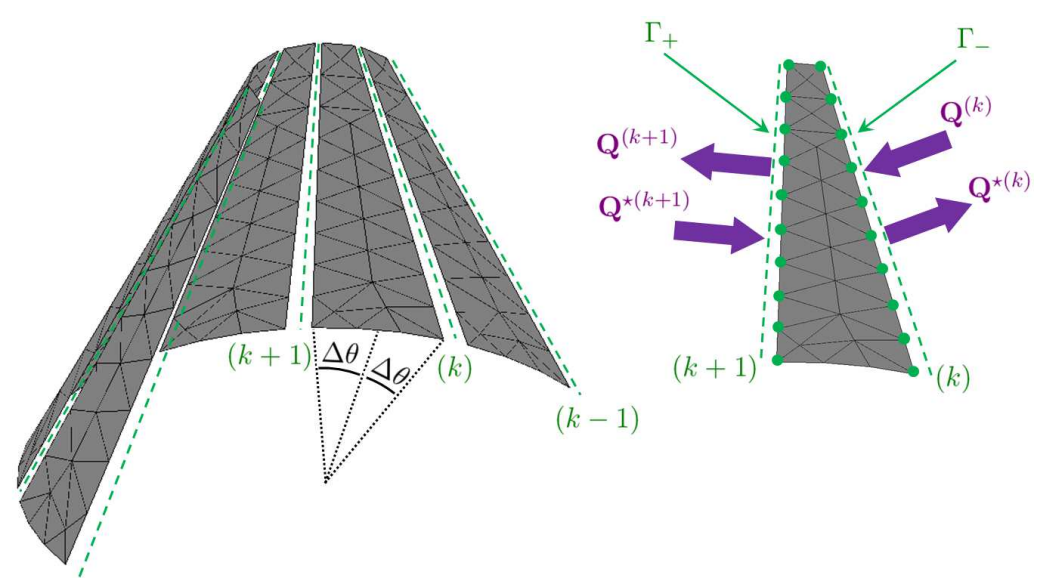

Figure 2: FE mesh of a periodic structure with a rotational periodicity of $2 \Delta \theta$ (left), and FE mesh of a substructure (right).

Consider a substructure which is enclosed between two interfaces $(k)$ and $(k+1)$ as shown in Figure 2, and denote as $\Gamma_{-}$and $\Gamma_{+}$the parts of the boundary of the substructure which match the interfaces $(k)$ and $(k+1)$, respectively. Also, assume that $\Gamma_{-}$and $\Gamma_{+}$are meshed in the same way with the same number $n$ of DOFs. In this case, the matrix $\mathbf{D}^{*}$ and the vectors $\mathbf{q}_{\Gamma}$ and $\mathbf{F}_{\Gamma}$ occurring in Eq. (5) may be expressed as:

$$
\mathbf{D}^{*}=\left[\begin{array}{ll}
\mathbf{D}_{\Gamma_{-} \Gamma_{-}}^{*} & \mathbf{D}_{\Gamma_{-} \Gamma_{+}}^{*} \\
\mathbf{D}_{\Gamma_{+} \Gamma_{-}}^{*} & \mathbf{D}_{\Gamma_{+} \Gamma_{+}}^{*}
\end{array}\right] \quad, \quad \mathbf{q}_{\Gamma}=\left[\begin{array}{c}
\mathbf{q}_{\Gamma_{-}}^{(k)} \\
\mathbf{q}_{\Gamma_{+}}^{(k+1)}
\end{array}\right] \quad, \quad \mathbf{F}_{\Gamma}=\left[\begin{array}{c}
\mathbf{F}_{\Gamma_{-}}^{(k)} \\
\mathbf{F}_{\Gamma_{+}}^{(k+1)}
\end{array}\right]
$$

where $\mathbf{D}_{\Gamma_{-} \Gamma_{-}}^{*}, \mathbf{D}_{\Gamma_{-} \Gamma_{+}}^{*}, \mathbf{D}_{\Gamma_{+} \Gamma_{-}}^{*}$ and $\mathbf{D}_{\Gamma_{+} \Gamma_{+}}^{*}$ are square matrices of same size $n \times n$. Also, $\mathbf{q}_{\Gamma_{-}}^{(k)}$, $\mathbf{q}_{\Gamma_{+}}^{(k+1)}, \mathbf{F}_{\Gamma_{-}}^{(k)}$ and $\mathbf{F}_{\Gamma_{+}}^{(k+1)}$ are vectors of same size $n \times 1$.

Eq. (5) is usually expressed in cartesian coordinates. However, within the framework of rotational periodicity, it has to be expressed in cylindrical coordinates, as follows:

$$
\widehat{\mathbf{D}}^{*} \widehat{\mathbf{q}}_{\Gamma}=\widehat{\mathbf{F}}_{\Gamma}
$$

where

$$
\begin{gathered}
\widehat{\mathbf{D}}^{*}=\mathcal{H} \mathbf{D}^{*} \mathcal{H}^{T} \quad, \quad \widehat{\mathbf{q}}_{\Gamma}=\mathcal{H} \mathbf{q}_{\Gamma} \quad, \quad \widehat{\mathbf{F}}_{\Gamma}=\mathcal{H} \mathbf{F}_{\Gamma}, \\
\mathcal{H}=\left[\begin{array}{cc}
\left(\mathbf{I}_{n / 3} \otimes \mathcal{T}_{-\Delta \theta}\right) & \mathbf{0} \\
\mathbf{0} & \left(\mathbf{I}_{n / 3} \otimes \mathcal{T}_{\Delta \theta}\right)
\end{array}\right] .
\end{gathered}
$$

Here, $\mathcal{T}_{\Delta \theta}$ and $\mathcal{T}_{-\Delta \theta}$ are two $3 \times 3$ orthogonal transformation matrices to link the unit vectors in cylindrical coordinates - say, $\left(\mathbf{e}_{r}, \mathbf{e}_{\theta}, \mathbf{e}_{z}\right)$ - to the unit vectors in cartesian coordinates $\left(\mathbf{e}_{x}, \mathbf{e}_{y}, \mathbf{e}_{z}\right)$ when $\theta=\Delta \theta$ and $\theta=-\Delta \theta$, respectively. Here, assumption is made that each node of the FE mesh of the substructure is modeled with 3 DOFs, and that the axis $\theta=0$ corresponds 
to the mid-plane (say, $(y, z)$ ) of the substructure. Also, in Eq. (9), $\otimes$ denotes the Kronecker product and $n / 3$ is the number of nodes on each interface $\Gamma_{+}$or $\Gamma_{-}$.

Eq. (7) may be rewritten in state vector form, as follows [18]:

$$
\left[\begin{array}{c}
\widehat{\mathbf{q}}_{\Gamma_{+}}^{(k+1)} \\
\widehat{\mathbf{F}}_{\Gamma_{+}}^{(k+1)}
\end{array}\right]=\widehat{\mathbf{S}}\left[\begin{array}{c}
\widehat{\mathbf{q}}_{\Gamma-}^{(k)} \\
-\widehat{\mathbf{F}}_{\Gamma_{-}}^{(k)}
\end{array}\right]=\widehat{\mathbf{S}}\left[\begin{array}{c}
\widehat{\mathbf{q}}_{\Gamma_{+}}^{(k)} \\
\widehat{\mathbf{F}}_{\Gamma_{+}}^{(k)}
\end{array}\right],
$$

where $\widehat{\mathrm{S}}$ is a $2 n \times 2 n$ symplectic matrix [18, 19], given by

$$
\widehat{\mathbf{S}}=\left[\begin{array}{cc}
-\widehat{\mathbf{D}}_{\Gamma_{-} \Gamma_{+}}^{*-1} \widehat{\mathbf{D}}_{\Gamma_{-} \Gamma_{-}}^{*} & -\widehat{\mathbf{D}}_{\Gamma_{-} \Gamma_{+}}^{*-1} \\
\widehat{\mathbf{D}}_{\Gamma_{+} \Gamma_{-}}^{*}-\widehat{\mathbf{D}}_{\Gamma_{+} \Gamma_{+}}^{*} \widehat{\mathbf{D}}_{\Gamma_{-} \Gamma_{+}}^{*-1} \widehat{\mathbf{D}}_{\Gamma_{-} \Gamma_{-}}^{*} & -\widehat{\mathbf{D}}_{\Gamma_{+} \Gamma_{+}}^{*} \widehat{\mathbf{D}}_{\Gamma_{-} \Gamma_{+}}^{*-1}
\end{array}\right] .
$$

To derive the last term in Eq. (10), the coupling conditions between two substructures have been considered, i.e., $\widehat{\mathbf{q}}_{\Gamma_{-}}^{(k)}=\widehat{\mathbf{q}}_{\Gamma_{+}}^{(k)}$ and $-\widehat{\mathbf{F}}_{\Gamma_{-}}^{(k)}=\widehat{\mathbf{F}}_{\Gamma_{+}}^{(k)}[20]$.

The transfer matrix between the substructures is given by $\widehat{\mathrm{S}}$, see Eq. (10). Its eigenvalues and eigenvectors are denoted as $\mu_{j}$ and $\phi_{j}$, respectively, and are referred to as the wave modes of the periodic structure. The eigenvalues $\mu_{j}$ have the meaning of wave parameters defined so that $\mu_{j}=\exp \left(-i \beta_{j} 2 \Delta \theta\right)$ where $\beta_{j}$ are circumferential wave numbers. Also, the eigenvectors $\phi_{j}$ denote vectors of wave shapes, which can be further partitioned as $\phi_{j}=\left[\phi_{\mathrm{q} j}^{T} \phi_{\mathrm{F} j}^{T}\right]^{T}$, where $\phi_{\mathrm{q} j}$ and $\phi_{\mathrm{F} j}$ are $n \times 1$ vectors of displacement and force components.

Since the matrix $\widehat{\mathbf{S}}$ is symplectic, its eigenvalues come in pairs as $\mu_{j}$ and $1 / \mu_{j}$. Also, since damping is taken into account in the modeling of the substructures, one has $\left|\mu_{j}\right| \neq 1$. As a rule of thumb, the eigensolutions of the matrix $\widehat{\mathbf{S}}$ may be classified into $n$ positive-going wave modes $\left(\mu_{j}, \phi_{j}\right)$ - i.e., those traveling around the positive circumferential direction - defined so that $\left|\mu_{j}\right|<1$, and $n$ negative-going wave modes $\left(\mu_{j}^{\star}, \phi_{j}^{\star}\right)$ defined so that $\mu_{j}^{\star}=1 / \mu_{j}$, i.e. $\left|\mu_{j}^{\star}\right|>1$.

\subsection{Forced response}

Consider a periodic structure with cyclic symmetry composed of $N$ substructures (see Figure 11), and $N$ interfaces $(k)$, with one excited substructure and other $N-1$ non-excited substructures. Assume, for the sake of clarity, that the excited substructure is enclosed between the interfaces $(N)$ and (1), and let us rewrite Eq. (4) in cylindrical coordinates, as follows:

$$
\widehat{\mathbf{D}}^{*}\left[\begin{array}{l}
\widehat{\mathbf{q}}_{\Gamma-}^{(N)} \\
\widehat{\mathbf{q}}_{\Gamma_{+}}^{(1)}
\end{array}\right]=\left[\begin{array}{c}
\widehat{\mathbf{F}}_{\Gamma_{-}}^{(N)} \\
\widehat{\mathbf{F}}_{\Gamma_{+}}^{(1)}
\end{array}\right]+\mathcal{H}\left(\mathcal{L}_{\Gamma \mathrm{B}}-\mathbf{D}_{\Gamma \mathrm{I}} \mathbf{D}_{\mathrm{II}}^{-1} \mathcal{L}_{\mathrm{IB}}\right) \mathbf{F}_{\mathrm{B}} .
$$

Within the WFE framework, the vectors $\widehat{\mathbf{q}}_{\Gamma_{-}}^{(1)}, \widehat{\mathbf{q}}_{\Gamma_{+}}^{(N)}, \widehat{\mathbf{F}}_{\Gamma_{-}}^{(1)}$ and $\widehat{\mathbf{F}}_{\Gamma_{+}}^{(N)}$ in Eq. (12) are to be expressed in terms of wave modes as follows [20]:

$$
\begin{aligned}
& \widehat{\mathbf{q}}_{\Gamma_{-}}^{(N)}=\boldsymbol{\Phi}_{\mathrm{q}} \boldsymbol{\mu}^{N-1} \mathbf{Q}+\boldsymbol{\Phi}_{\mathrm{q}}^{\star} \mathbf{Q}^{\star} \quad, \quad \widehat{\mathbf{q}}_{\Gamma_{+}}^{(1)}=\boldsymbol{\Phi}_{\mathrm{q}} \mathbf{Q}+\boldsymbol{\Phi}_{\mathrm{q}}^{\star} \boldsymbol{\mu}^{N-1} \mathbf{Q}^{\star}, \\
& -\widehat{\mathbf{F}}_{\Gamma_{-}}^{(N)}=\boldsymbol{\Phi}_{\mathrm{F}} \boldsymbol{\mu}^{N-1} \mathbf{Q}+\boldsymbol{\Phi}_{\mathrm{F}}^{\star} \mathbf{Q}^{\star} \quad, \quad \widehat{\mathbf{F}}_{\Gamma_{+}}^{(1)}=\boldsymbol{\Phi}_{\mathrm{F}} \mathbf{Q}+\boldsymbol{\Phi}_{\mathrm{F}}^{\star} \boldsymbol{\mu}^{N-1} \mathbf{Q}^{\star},
\end{aligned}
$$

where $\Phi_{\mathrm{q}}=\left[\phi_{\mathrm{q} 1} \cdots \phi_{\mathrm{q} n}\right], \Phi_{\mathrm{q}}^{\star}=\left[\phi_{\mathrm{q} 1}^{\star} \cdots \phi_{\mathrm{q} n}^{\star}\right], \boldsymbol{\Phi}_{\mathrm{F}}=\left[\phi_{\mathrm{F} 1} \cdots \phi_{\mathrm{F} n}\right]$ and $\boldsymbol{\Phi}_{\mathrm{F}}^{\star}=\left[\phi_{\mathrm{F} 1}^{\star} \cdots \phi_{\mathrm{F} n}^{\star}\right]$ are $n \times n$ matrices of wave shapes. Also, $\boldsymbol{\mu}=\operatorname{diag}\left\{\mu_{j}\right\}_{j}$ is the $n \times n$ diagonal matrix of the 
eigenvalues of $\widehat{\mathbf{S}}$ that concern the positive-going wave modes, i.e., those for which $\left|\mu_{j}\right|<1$. Finally, $\mathbf{Q}=\mathbf{Q}^{(1)}$ and $\mathbf{Q}^{\star}=\mathbf{Q}^{\star(N)}$ are $n \times 1$ vectors of wave amplitudes, expressed at the "right" and "left" sides of the excited substructure, see Figure 3 .

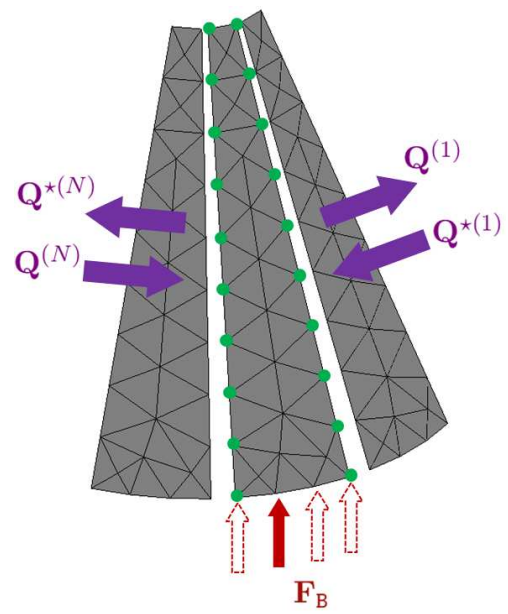

Figure 3: Vectors of wave amplitudes $\mathbf{Q}^{(1)}, \mathbf{Q}^{\star(1)}, \mathbf{Q}^{(N)}$ and $\mathbf{Q}^{\star(N)}$ at the coupling interfaces of the excited substructure.

Eq. (12) leads to

$\widehat{\mathbf{D}}^{*}\left[\begin{array}{cc}\boldsymbol{\Phi}_{\mathrm{q}} \boldsymbol{\mu}^{N-1} & \boldsymbol{\Phi}_{\mathrm{q}}^{\star} \\ \boldsymbol{\Phi}_{\mathrm{q}} & \boldsymbol{\Phi}_{\mathrm{q}}^{\star} \boldsymbol{\mu}^{N-1}\end{array}\right]\left[\begin{array}{c}\mathrm{Q} \\ \mathbf{Q}^{\star}\end{array}\right]=\left[\begin{array}{cc}-\boldsymbol{\Phi}_{\mathrm{F}} \boldsymbol{\mu}^{N-1} & -\boldsymbol{\Phi}_{\mathrm{F}}^{\star} \\ \boldsymbol{\Phi}_{\mathrm{F}} & \boldsymbol{\Phi}_{\mathrm{F}}^{\star} \boldsymbol{\mu}^{N-1}\end{array}\right]\left[\begin{array}{c}\mathbf{Q} \\ \mathbf{Q}^{\star}\end{array}\right]+\mathcal{H}\left(\mathcal{L}_{\Gamma \mathrm{B}}-\mathbf{D}_{\Gamma \mathrm{I}} \mathbf{D}_{\mathrm{II}}^{-1} \mathcal{L}_{\mathrm{IB}}\right) \mathbf{F}_{\mathrm{B}}$.

Eq. (15) may be further simplified to give:

$$
\left(\widehat{\mathbf{D}}^{*} \boldsymbol{\Psi}_{\mathrm{q}}-\boldsymbol{\Psi}_{\mathrm{F}}\right)\left[\begin{array}{cc}
\mathbf{0} & \mathbf{I}_{n}-\boldsymbol{\mu}^{N} \\
\mathbf{I}_{n}-\boldsymbol{\mu}^{N} & \mathbf{0}
\end{array}\right]\left[\begin{array}{c}
\mathbf{Q} \\
\mathbf{Q}^{\star}
\end{array}\right]=\mathcal{H}\left(\mathcal{L}_{\Gamma \mathrm{B}}-\mathbf{D}_{\Gamma \mathrm{I}} \mathbf{D}_{\mathrm{II}}^{-1} \mathcal{L}_{\mathrm{IB}}\right) \mathbf{F}_{\mathrm{B}},
$$

where

$$
\Psi_{\mathrm{q}}=\left[\begin{array}{cc}
\Phi_{\mathrm{q}}^{\star} & 0 \\
0 & \boldsymbol{\Phi}_{\mathrm{q}}
\end{array}\right] \quad, \quad \boldsymbol{\Psi}_{\mathrm{F}}=\left[\begin{array}{cc}
-\boldsymbol{\Phi}_{\mathrm{F}}^{\star} & 0 \\
0 & \boldsymbol{\Phi}_{\mathrm{F}}
\end{array}\right]
$$

To derive Eq. (16), the following properties of wave modes, which result from the eigenproblem (10), have been considered:

$$
\widehat{D}^{*}\left[\begin{array}{c}
\Phi_{\mathrm{q}} \\
\Phi_{\mathrm{q}} \boldsymbol{\mu}
\end{array}\right]=\left[\begin{array}{c}
-\Phi_{\mathrm{F}} \\
\boldsymbol{\Phi}_{\mathrm{F}} \boldsymbol{\mu}
\end{array}\right] \quad, \quad \widehat{\mathrm{D}}^{*}\left[\begin{array}{c}
\Phi_{\mathrm{q}}^{\star} \boldsymbol{\mu} \\
\boldsymbol{\Phi}_{\mathrm{q}}^{\star}
\end{array}\right]=\left[\begin{array}{c}
-\boldsymbol{\Phi}_{\mathrm{F}}^{\star} \boldsymbol{\mu} \\
\boldsymbol{\Phi}_{\mathrm{F}}^{\star}
\end{array}\right]
$$

Solving Eq. (16) yields the vectors of wave amplitudes $\mathrm{Q}$ and $\mathrm{Q}^{\star}$, i.e.:

$$
\left[\begin{array}{c}
\mathbf{Q} \\
\mathbf{Q}^{\star}
\end{array}\right]=\left[\begin{array}{cc}
\mathbf{0} & \left(\mathbf{I}_{n}-\boldsymbol{\mu}^{N}\right)^{-1} \\
\left(\mathbf{I}_{n}-\boldsymbol{\mu}^{N}\right)^{-1} & \mathbf{0}
\end{array}\right]\left(\widehat{\mathbf{D}}^{*} \Psi_{\mathrm{q}}-\mathbf{\Psi}_{\mathrm{F}}\right)^{-1} \mathcal{H}\left(\mathcal{L}_{\Gamma \mathrm{B}}-\mathbf{D}_{\Gamma \mathrm{I}} \mathbf{D}_{\mathrm{II}}^{-1} \mathcal{L}_{\mathrm{IB}}\right) \mathbf{F}_{\mathrm{B}} .
$$

The determination of the vectors of nodal displacements and nodal forces on the coupling interfaces, between the substructures, follows as:

$$
\begin{gathered}
\widehat{\mathbf{q}}_{\Gamma_{+}}^{(k)}=\widehat{\mathbf{q}}_{\Gamma_{-}}^{(k)}=\boldsymbol{\Phi}_{\mathrm{q}} \boldsymbol{\mu}^{k-1} \mathbf{Q}+\boldsymbol{\Phi}_{\mathrm{q}}^{\star} \boldsymbol{\mu}^{N-k} \mathbf{Q}^{\star}, \\
\widehat{\mathbf{F}}_{\Gamma_{+}}^{(k)}=-\widehat{\mathbf{F}}_{\Gamma_{-}}^{(k)}=\boldsymbol{\Phi}_{\mathrm{F}} \boldsymbol{\mu}^{k-1} \mathbf{Q}+\boldsymbol{\Phi}_{\mathrm{F}}^{\star} \boldsymbol{\mu}^{N-k} \mathbf{Q}^{\star} .
\end{gathered}
$$




\subsection{Dynamic flexibility modes}

Recall that the periodic structure under concern is made up of $N$ substructures with one excited substructure - i.e., the one enclosed between the interfaces $(N)$ and (1) (see Figure 3) - , the other being free of external excitations. The displacement vector of a non-excited substructure enclosed between two interfaces $(k)$ and $(k+1)(k=1, \cdots, N-1)$, expressed in its local cartesian frame, is given by:

$$
\left[\begin{array}{l}
\mathbf{q}_{\Gamma} \\
\mathbf{q}_{\mathrm{I}}
\end{array}\right]=\left[\begin{array}{c}
\mathbf{I}_{2 n} \\
-\mathbf{D}_{\mathrm{II}}^{-1} \mathbf{D}_{\mathrm{I} \Gamma}
\end{array}\right] \mathbf{q}_{\Gamma}=\left[\begin{array}{c}
\mathbf{I}_{2 n} \\
-\mathbf{D}_{\mathrm{II}}^{-1} \mathbf{D}_{\mathrm{I} \Gamma}
\end{array}\right]\left[\begin{array}{c}
\mathbf{q}_{\Gamma-}^{(k)} \\
\mathbf{q}_{\Gamma_{+}}^{(k+1)}
\end{array}\right]
$$

In this case, the displacement vector for the internal DOFs is given by $\mathbf{q}_{\mathrm{I}}=-\mathbf{D}_{\mathrm{II}}^{-1} \mathbf{D}_{\mathrm{I \Gamma}} \mathbf{q}_{\Gamma}$, as a consequence of Eq. (1) when $\mathbf{F}_{\mathrm{I}}=0$. From Eqs. (8) and (20), Eq. (22) leads to

$$
\left[\begin{array}{l}
\mathbf{q}_{\Gamma} \\
\mathbf{q}_{\mathrm{I}}
\end{array}\right]=\left[\begin{array}{c}
\mathbf{I}_{2 n} \\
-\mathbf{D}_{\mathrm{II}}^{-1} \mathbf{D}_{\mathrm{I} \Gamma}
\end{array}\right] \mathcal{H}^{T}\left[\begin{array}{cc}
\boldsymbol{\Phi}_{\mathrm{q}} \boldsymbol{\mu}^{k-1} & \boldsymbol{\Phi}_{\mathrm{q}}^{\star} \boldsymbol{\mu}^{N-k} \\
\boldsymbol{\Phi}_{\mathrm{q}} \boldsymbol{\mu}^{k} & \boldsymbol{\Phi}_{\mathrm{q}}^{\star} \boldsymbol{\mu}^{N-k-1}
\end{array}\right]\left[\begin{array}{c}
\mathbf{Q} \\
\mathbf{Q}^{\star}
\end{array}\right] .
$$

In the same way as Eq. (23), the displacement vector of the excited substructure, for the interface and internal DOFs, may be expressed as:

$$
\left[\begin{array}{l}
\mathbf{q}_{\Gamma} \\
\mathbf{q}_{\mathrm{I}}
\end{array}\right]=\left[\begin{array}{c}
\mathbf{I}_{2 n} \\
-\mathbf{D}_{\mathrm{II}}^{-1} \mathbf{D}_{\mathrm{I} \Gamma}
\end{array}\right] \mathcal{H}^{T}\left[\begin{array}{cc}
\boldsymbol{\Phi}_{\mathrm{q}} \boldsymbol{\mu}^{N-1} & \boldsymbol{\Phi}_{\mathrm{q}}^{\star} \\
\boldsymbol{\Phi}_{\mathrm{q}} & \boldsymbol{\Phi}_{\mathrm{q}}^{\star} \boldsymbol{\mu}^{N-1}
\end{array}\right]\left[\begin{array}{c}
\mathbf{Q} \\
\mathbf{Q}^{\star}
\end{array}\right]+\left[\begin{array}{c}
\mathbf{0} \\
\mathbf{D}_{\mathrm{II}}^{-1} \mathcal{L}_{\mathrm{IB}}
\end{array}\right] \mathbf{F}_{\mathrm{B}} .
$$

Let us define as $\mathbf{q}^{(k)}$ the displacement vector that concerns the interface DOFs on $\Gamma_{-}$and the internal DOFs of a substructure enclosed between two arbitrary interfaces $(k)$ and $(k+1)$ $(k=1, \cdots, N$, with $(N+1)=(1))$, as follows:

$$
\mathbf{q}^{(k)}=\left[\begin{array}{l}
\mathbf{q}_{\Gamma-}^{(k)} \\
\mathbf{q}_{\mathrm{I}}^{(k)}
\end{array}\right]
$$

$\mathbf{q}^{(k)}$ is simply obtained either from Eq. (23) or Eq. (24) by removing the second row block which is related to the interface DOFs on $\Gamma_{+}$. The size of the vector $\mathbf{q}^{(k)}$ is $\left(n+n_{\mathrm{I}}\right) \times 1$, where $n$ is the number of DOFs on $\Gamma_{-}$and $n_{\mathrm{I}}$ is the number of internal DOFs. In this way, by considering all the displacement vectors $\mathbf{q}^{(k)}$ of the substructures, the whole displacement vector of the periodic structure may be simply built.

Notice that the displacement vectors $\mathbf{q}^{(k)}$ of the substructures are to be expressed in the global cartesian coordinate system of the periodic structure. As a whole, the displacement vector of the periodic structure may be built as follows:

$$
\mathbf{q}=\sum_{k=1}^{N} \mathcal{L}^{(k) T}\left(\mathbf{I}_{\left(n+n_{\mathrm{I}}\right) / 3} \otimes \mathcal{R}_{2 \Delta \theta}^{k}\right) \mathbf{q}^{(k)}=\left[\begin{array}{c}
\left(\mathbf{I}_{\left(n+n_{\mathrm{I}}\right) / 3} \otimes \mathcal{R}_{2 \Delta \theta}\right) \mathbf{q}^{(1)} \\
\left(\mathbf{I}_{\left(n+n_{\mathrm{I}}\right) / 3} \otimes \mathcal{R}_{2 \Delta \theta}^{2}\right) \mathbf{q}^{(2)} \\
\vdots \\
\left(\mathbf{I}_{\left(n+n_{\mathrm{I}}\right) / 3} \otimes \mathcal{R}_{2 \Delta \theta}^{N}\right) \mathbf{q}^{(N)}
\end{array}\right]
$$

where $\mathcal{L}^{(k)}$ are Boolean localization matrices and $\mathcal{R}_{2 \Delta \theta}$ is a rotation matrix defined so that $\mathcal{R}_{2 \Delta \theta}^{N}=\mathbf{I}_{3}$.

For each state of excitation $i$ for which one boundary DOF $i$ of a substructure is excited by a unit force (see Figure 3), there corresponds a so-called dynamic flexibility mode $\mathbf{q}_{i}=\chi_{i}$ which 
is given by Eq. (26). In fact, there are as many dynamic flexibility modes as the number of boundary DOFs of the structure. To determine all the dynamic flexibility modes of the whole periodic structure, all the states of excitations of an excited substructure should be analyzed, but not only since all the substructures have to be scanned as well.

Assume, as was previously made, that the excited substructure is the one enclosed between the interfaces $(N)$ and $(1)$. Hence, a matrix of dynamic flexibility modes may be formulated which concerns the different states of excitations for the boundary DOFs of the substructure (see Figure 4), as follows:

$$
\mathbf{X}^{(N)}=\left[\chi_{1}^{(N)} \chi_{2}^{(N)} \cdots\right] .
$$

As a result, the displacement vector of the periodic structure which results from an arbitrary vector of boundary forces $\mathbf{F}_{\mathrm{B}}^{(N)}$ applied to the substructure enclosed between the interfaces $(N)$ and (1), is given by:

$$
\mathbf{q}=\mathbf{X}^{(N)} \mathbf{F}_{\mathrm{B}}^{(N)}
$$

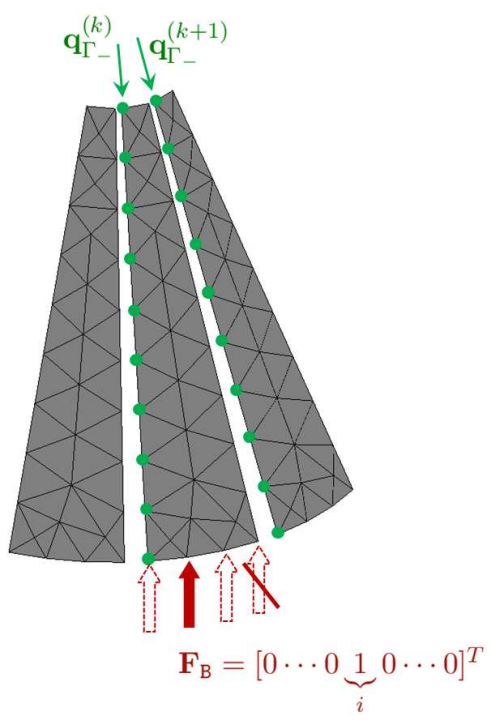

Figure 4: Illustration of the states of excitations which need to be considered for each substructure to determine the dynamic flexibility modes of the periodic structure.

Concerning an excited substructure enclosed between two arbitrary interfaces $(k)$ and $(k+$ $1)$. The matrix of dynamic flexibility modes can be simply derived from $\mathbf{X}^{(N)}$ through cyclic permutation, as follows:

$$
\mathbf{q} \rightarrow\left(\mathbf{I}_{n_{\mathrm{T}} / 3} \otimes \mathcal{R}_{2 \Delta \theta}^{k}\right) \mathcal{P}^{(k)} \mathbf{q}=\left(\mathbf{I}_{n_{\mathrm{T}} / 3} \otimes \mathcal{R}_{2 \Delta \theta}^{k}\right)\left[\begin{array}{c}
\left(\mathbf{I}_{\left(n+n_{\mathrm{I}}\right) / 3} \otimes \mathcal{R}_{2 \Delta \theta}^{N-k+1}\right) \mathbf{q}^{(N-k+1)} \\
\vdots \\
\left(\mathbf{I}_{\left(n+n_{\mathrm{I}}\right) / 3} \otimes \mathcal{R}_{2 \Delta \theta}^{N}\right) \mathbf{q}^{(N)} \\
\left(\mathbf{I}_{\left(n+n_{\mathrm{I}}\right) / 3} \otimes \mathcal{R}_{2 \Delta \theta}\right) \mathbf{q}^{(1)} \\
\vdots \\
\left(\mathbf{I}_{\left(n+n_{\mathrm{I}}\right) / 3} \otimes \mathcal{R}_{2 \Delta \theta}^{N-k}\right) \mathbf{q}^{(N-k)}
\end{array}\right]
$$

where $n_{\mathrm{T}}$ is the number of DOFs of the whole periodic structure. Also, the vector of boundary forces of the substructure should be expressed as follows:

$$
\mathbf{F}_{\mathrm{B}}^{(k)}=\left(\mathbf{I}_{n_{\mathrm{B}} / 3} \otimes \mathcal{R}_{2 \Delta \theta}^{k}\right)^{T} \mathbf{F}_{\mathrm{B}}^{(N)},
$$


where $n_{\mathrm{B}}$ is the number of boundary DOFs of each substructure with the exception of those on $\Gamma_{+}$. Eqs. (29) and (30) yield the following matrix of dynamic flexibility modes:

$$
\mathbf{X}^{(k)}=\left(\mathbf{I}_{n_{\mathrm{T}} / 3} \otimes \mathcal{R}_{2 \Delta \theta}^{k}\right) \mathcal{P}^{(k)} \mathbf{X}^{(N)}\left(\mathbf{I}_{n_{\mathrm{B}} / 3} \otimes \mathcal{R}_{2 \Delta \theta}^{k}\right) .
$$

Hence, the matrix of dynamic flexibility modes of the whole periodic structure may be written as:

$$
\mathbf{X}=\left[\mathbf{X}^{(1)} \mathbf{X}^{(2)} \cdots \mathbf{X}^{(N)}\right]
$$

\subsection{Domain decomposition}

Consider, for the sake of simplicity, two periodic structures 1 and 2 which are connected to each other as shown in Figure 5. Also, assume that the FE meshes of the structures are compatible across the coupling interface, i.e., regarding the boundary DOFs where the structures are coupled together.
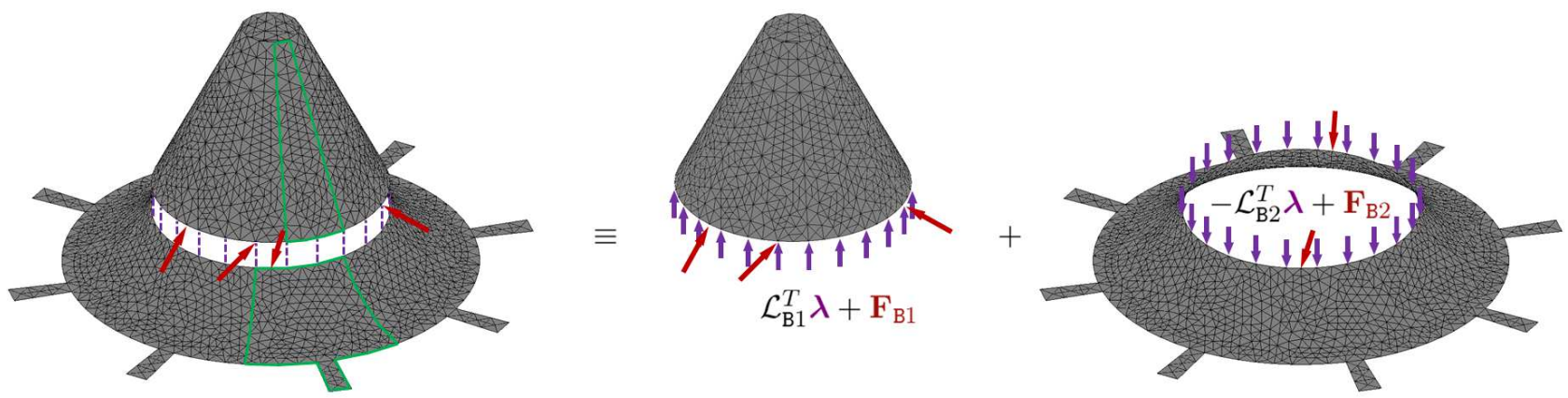

Figure 5: FE meshes of two periodic structures which are connected to each other and which are subject to external forces on their boundaries.

Denote as $\boldsymbol{\lambda}$ the vector of coupling forces and denote as $\mathbf{F}_{\mathrm{B} 1}$ and $\mathbf{F}_{\mathrm{B} 2}$ the vectors of external forces which may be considered on the boundaries of the periodic structures 1 and 2, respectively. Hence, the vectors of boundary forces may be defined as $\mathbf{F}_{\mathrm{B} 1}+\mathcal{L}_{\mathrm{B} 1}^{T} \boldsymbol{\lambda}$ and $\mathbf{F}_{\mathrm{B} 2}-\mathcal{L}_{\mathrm{B} 2}^{T} \boldsymbol{\lambda}$ (Figure 5) where $\mathcal{L}_{\mathrm{B} 1}$ and $\mathcal{L}_{\mathrm{B} 2}$ are Boolean matrices which localize the coupling DOFs among the boundary DOFs of the periodic structures 1 and 2, respectively.

By considering the matrices of dynamic flexibility modes of the periodic structures - namely, $\mathbf{X}_{1}$ and $\mathbf{X}_{2}$-, the displacement vectors of the periodic structures - namely, $\mathbf{q}_{1}$ and $\mathbf{q}_{2}-$ may be expressed as follows:

$$
\mathbf{q}_{1}=\mathbf{X}_{1}\left(\mathbf{F}_{\mathrm{B} 1}+\mathcal{L}_{\mathrm{B} 1}^{T} \boldsymbol{\lambda}\right) \quad, \quad \mathbf{q}_{2}=\mathbf{X}_{2}\left(\mathbf{F}_{\mathrm{B} 2}-\mathcal{L}_{\mathrm{B} 2}^{T} \boldsymbol{\lambda}\right) .
$$

On the other hand, the kinematic constraints on the coupling interface are given by:

$$
\mathcal{L}_{1} \mathbf{q}_{1}-\mathcal{L}_{2} \mathbf{q}_{2}=\mathbf{0},
$$

where $\mathcal{L}_{1}$ and $\mathcal{L}_{2}$ are Boolean matrices to localize the coupling DOFs among all the DOFs of the structures 1 and 2, respectively. Eqs. (33) and (34) lead to the following matrix equation:

$$
\left[\begin{array}{ccc}
\mathbf{I} & \mathbf{0} & -\mathbf{X}_{1} \mathcal{L}_{\mathrm{B} 1}^{T} \\
\mathbf{0} & \mathbf{I} & \mathbf{X}_{2} \mathcal{L}_{\mathrm{B} 2}^{T} \\
\mathcal{L}_{1} & -\mathcal{L}_{2} & \mathbf{0}
\end{array}\right]\left[\begin{array}{c}
\mathbf{q}_{1} \\
\mathbf{q}_{2} \\
\boldsymbol{\lambda}
\end{array}\right]=\left[\begin{array}{c}
\mathbf{X}_{1} \mathbf{F}_{\mathrm{B} 1} \\
\mathbf{X}_{2} \mathbf{F}_{\mathrm{B} 2} \\
\mathbf{0}
\end{array}\right]
$$


Solving Eq. (35) yields:

$$
\boldsymbol{\lambda}=-\left(\mathcal{L}_{1} \mathbf{X}_{1} \mathcal{L}_{\mathrm{B} 1}^{T}+\mathcal{L}_{2} \mathbf{X}_{2} \mathcal{L}_{\mathrm{B} 2}^{T}\right)^{-1}\left(\mathcal{L}_{1} \mathbf{X}_{1} \mathbf{F}_{\mathrm{B} 1}-\mathcal{L}_{2} \mathbf{X}_{2} \mathbf{F}_{\mathrm{B} 2}\right) .
$$

The displacement vectors of the periodic structures follow from Eq. (33), i.e.:

$$
\mathbf{q}_{1}=\mathbf{X}_{1}\left(\mathbf{F}_{\mathrm{B} 1}+\mathcal{L}_{\mathrm{B} 1}^{T} \boldsymbol{\lambda}\right) \quad, \quad \mathbf{q}_{2}=\mathbf{X}_{2}\left(\mathbf{F}_{\mathrm{B} 2}-\mathcal{L}_{\mathrm{B} 2}^{T} \boldsymbol{\lambda}\right)
$$

The analysis of assemblies made up of three of more periodic structures can be achieved through simple adaptations of the proposed strategy.

\section{NUMERICAL RESULTS}

\subsection{Single periodic structure}

Consider first a single periodic structure as shown in Figure 6, with $N=36$ substructures. Each substructure is meshed using four node tetrahedral elements, with three translational DOFs per node, leading to 3786 DOFs and $n=84$ DOFs on each substructure interface (either $\Gamma_{+}$or $\left.\Gamma_{-}\right)$. The boundary DOFs of the periodic structure, i.e., where external excitations may occur, are those located on the inner circumferential surface (see Figure 6). The boundary DOFs which are considered to model the substructures (see Section 2.4) are highlighted in red spots in Figure 6

The material properties of the periodic structure are: density of $7800 \mathrm{~kg} / \mathrm{m}^{3}$, Young's modulus of $2.1 \times 10^{11} \mathrm{~Pa}$, Poisson's ratio of 0.3 and loss factor of $5 \times 10^{-3}$. The periodic structure is excited by means of four pairs of point forces acting along the circumferential direction, at $\theta=0^{\circ}, \theta=90^{\circ}, \theta=180^{\circ}$ and $\theta=270^{\circ}$ on the inner circumferential boundary as shown in Figure 6 ,

Here, the magnitude of the transverse displacement ( $x$-direction) of a given substructure is analyzed at one measurement point as shown in Figure 6. Hence, the frequency response function (FRF) of the periodic structure is analyzed which is done by considering a frequency band of $[0 \mathrm{~Hz}, 2500 \mathrm{~Hz}]$, say, by considering a sample of 500 discrete frequencies which are equally spaced with a frequency step of $5 \mathrm{~Hz}$.

\subsubsection{Comparison with the FE method}

The FRFs of the periodic structure which are issued from the FE method and the proposed approach are shown in Figure 7. Both FRFs have been calculated using MATLAB ${ }^{\circledR}$ and the same processor $\left(\right.$ Intel $^{\circledR}$ Core $^{\mathrm{TM}}$ i7-3720QM). Notice that the condensed dynamic stiffness matrices of the substructures (see after Eq. (4)) have been considered when implementing the proposed approach and the FE method. Recall that the condensed dynamic stiffness matrices of the substructures are identical, i.e., only one condensed dynamic stiffness matrix of a substructure needs to be computed.

Regarding Figure 7, it is seen that the solution issued from the proposed approach is in perfect agreement with the FE method. In fact, the mean relative error between the two FRFs may be assessed as $<\left|q_{\mathrm{WFE}}^{\text {mes }}-q_{\mathrm{FE}}^{\text {mes }}\right| /\left|q_{\mathrm{FE}}^{\text {mes }}\right|>_{f}$, where $q^{\text {mes }}$ is the measured displacement and $<\bullet>_{f}$ represents the average over the frequency domain. Here, it is about $7 \times 10^{-6 \%}$, say, very small. 


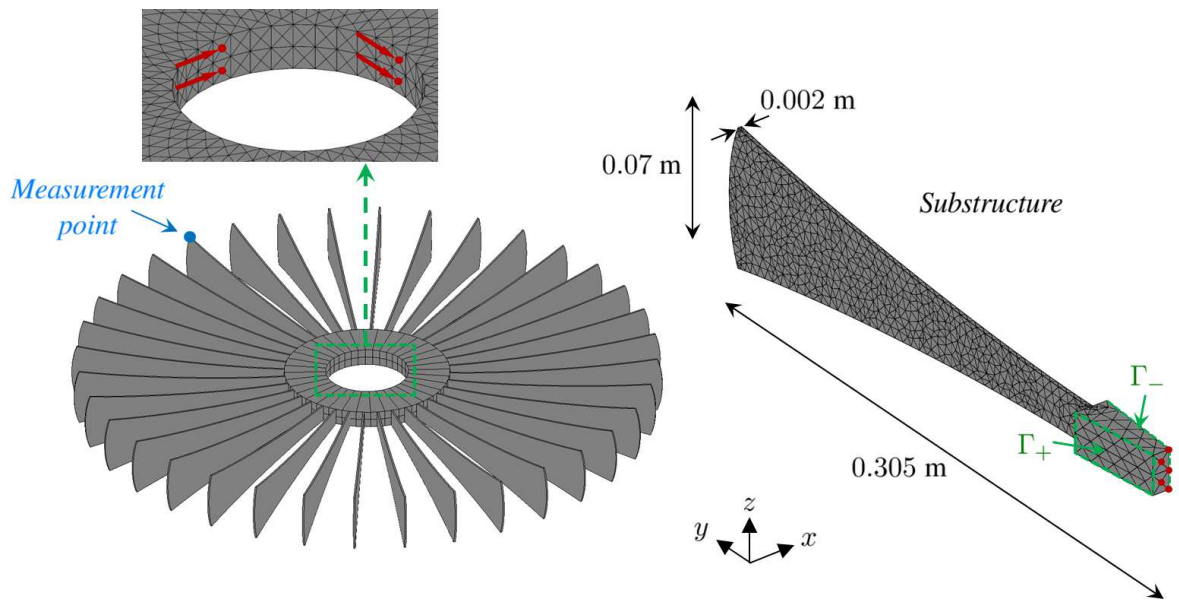

Figure 6: Periodic structure with 36 substructures (left), and FE mesh of a substructure (right); (red spots) boundary DOFs of the substructures.

In terms of CPU times, it appears that the FE method works better than the proposed approach, i.e., $3300 \mathrm{~s}$ against $4800 \mathrm{~s}$ to compute the FRF. The apparent efficiency of the FE method lies in the fact that a moderate number of substructures, i.e., $N=36$, have been considered to model the periodic structure. In this case, the computational cost involved for assembling those 36 substructures, with the FE method, is not excessive. The computational burden of the FE method is likely to increase as soon as the number of substructures increases, as well as the number of periodic structures involved (see next subsection).

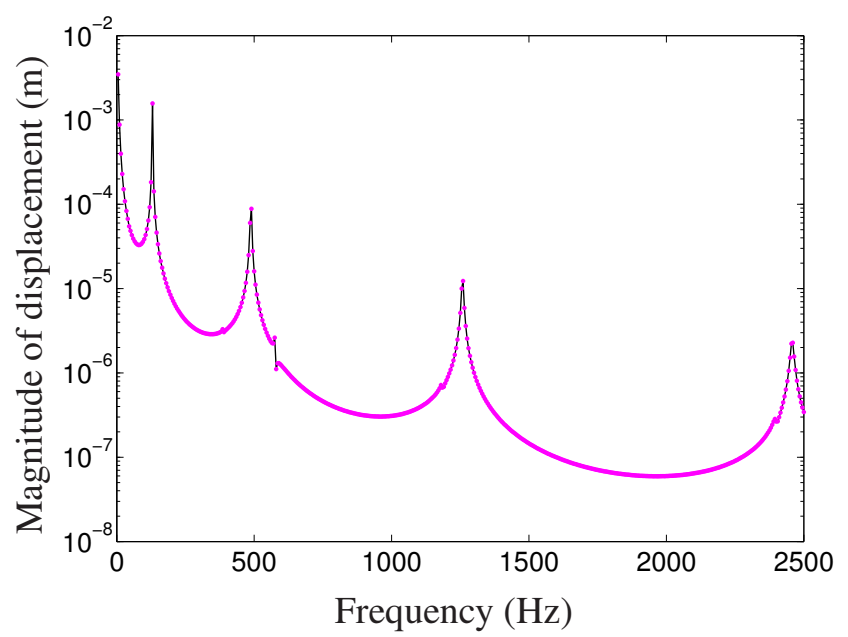

Figure 7: FRF of the periodic structure with 36 substructures: (black solid line) FE method; (pink dots) proposed approach.

\subsubsection{Comparison with the theory of cyclic symmetry}

Also, the theory of cyclic symmetry is considered. It involves considering a discrete Fourier transform [2] of the dynamic stiffness matrix of the periodic structure, and reducing it accordingly to obtain a set of small uncoupled matrix systems (see [2, 21] for further details). The FRF issued from the theory of cyclic symmetry is displayed in Figure 8 along with the FE solution. It is seen that the two solutions perfectly match to each other. In this case, the mean relative error, 
say $<\left|q_{\mathrm{CYC}}^{\mathrm{mes}}-q_{\mathrm{FE}}^{\mathrm{mes}}\right| /\left|q_{\mathrm{FE}}^{\mathrm{mes}}\right|>_{f}$, is about $13 \times 10^{-6 \%}$, i.e., it is of the same order as the proposed approach. The CPU times involved in the theory of cyclic symmetry is about 2200 s, i.e., it is small compared to the proposed approach. Here again, the efficiency of the proposed approach is questionable. Keep in mind, however, that the theory of cyclic symmetry may suffer from a lack of accuracy for modeling assemblies made up of several periodic structures, as explained in Introduction (Section 1). This is not the case with the proposed approach which is a priori exact, as explained in the next subsection.

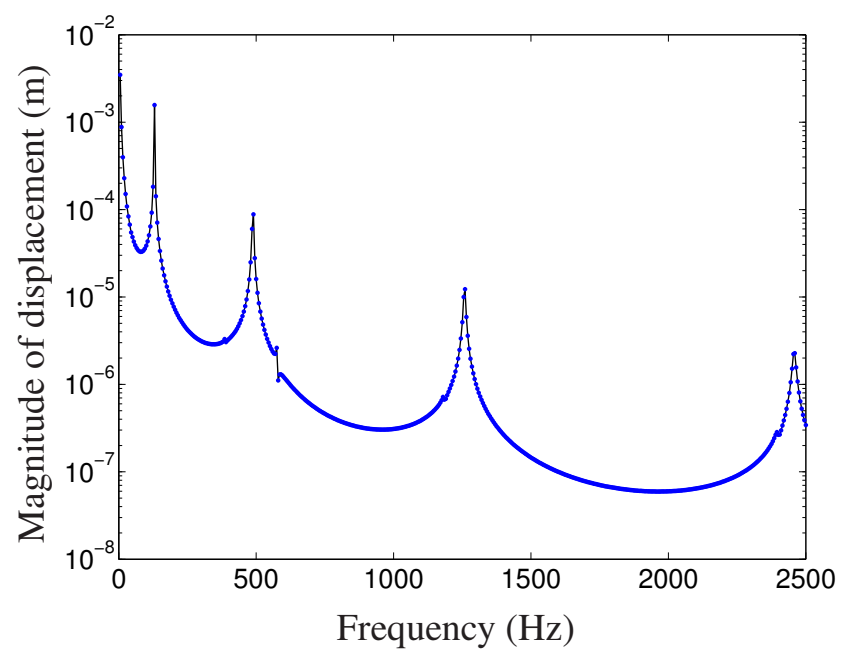

Figure 8: FRF of the periodic structure with 36 substructures: (black solid line) FE method; (blue dots) theory of cyclic symmetry.

\subsection{Two periodic structures}

Consider now the case where the previous periodic structure is coupled on part of its boundary with a second periodic structure with 60 substructures as shown in Figure 9. The material properties of the second periodic structure are similar to those of the previous one. Concerning this second periodic structure, 1746 DOFs and 117 DOFs are respectively used to mesh each substructure and each interface. The two periodic structures are coupled on twelve nodes which are periodically distributed around their inner circumferential surfaces as shown in Figure 9 in red dashed lines, where the continuity of the displacements about the $x, y$ and $z$ directions is assumed.

\subsubsection{Comparison with the FE method}

The FRFs of the structural assembly are shown in Figure 10. Again, the accuracy of the proposed approach can be highlighted without any ambiguity, the mean relative error between the two FRFs being $0.4 \%$. Also, in this case, the computational efficiency of the proposed method is better than the FE method, i.e., $7700 \mathrm{~s}$ against 15, $500 \mathrm{~s}$ to compute the FRF of the whole structure. This means $50 \%$ time saving in benefit of the proposed approach. The computational burden of the FE method is mainly due to the computation of the dynamic stiffness matrix of the whole structure, which requires one to (i) build the dynamic stiffness matrices of the periodic structures, separately, from the condensed dynamic stiffness matrices of the substructures, and 

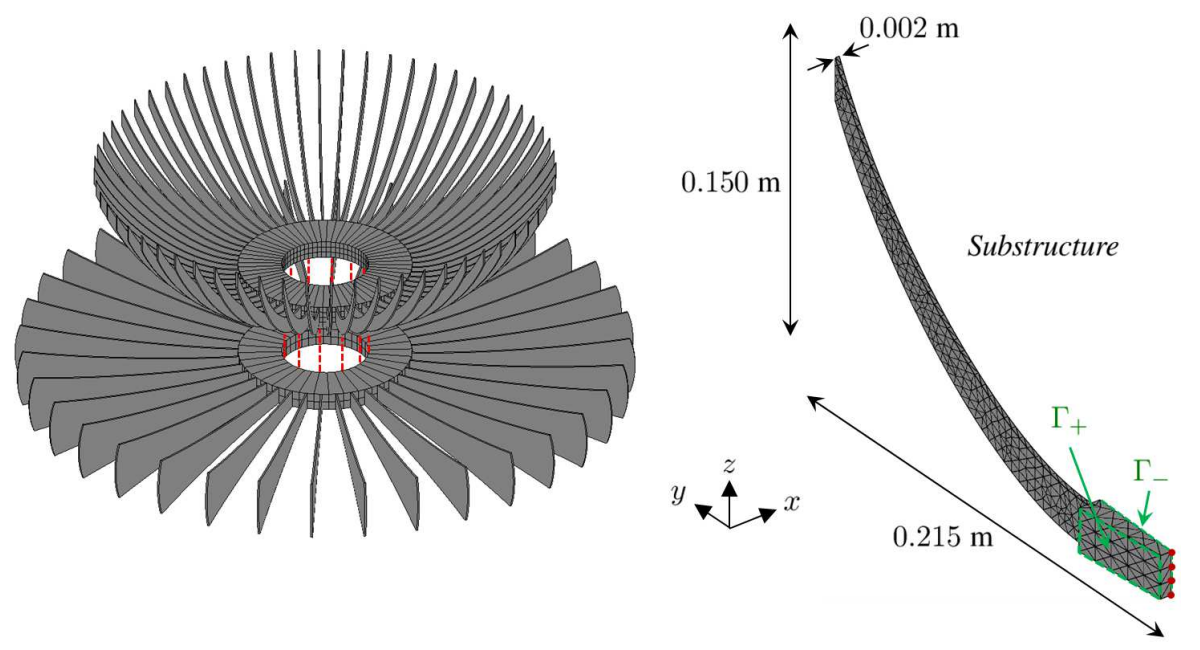

Figure 9: Assembly made up of two periodic structures with 36 and 60 substructures, respectively (left), and FE mesh of a substructure composing the periodic structure with 60 substructures (right).

(ii) assemble these by considering the displacement compatibility conditions at the coupling nodes. In comparison, the proposed approach involves building matrices of dynamic flexibility modes, which is quickly done by considering the periodic nature of the structures; notice that this task is almost insensitive to the number of substructures considered. Also, the matrices of dynamic flexibility modes are rectangular matrices whose column size is not excessive, i.e., the construction of the matrix system (35) can be quickly achieved.

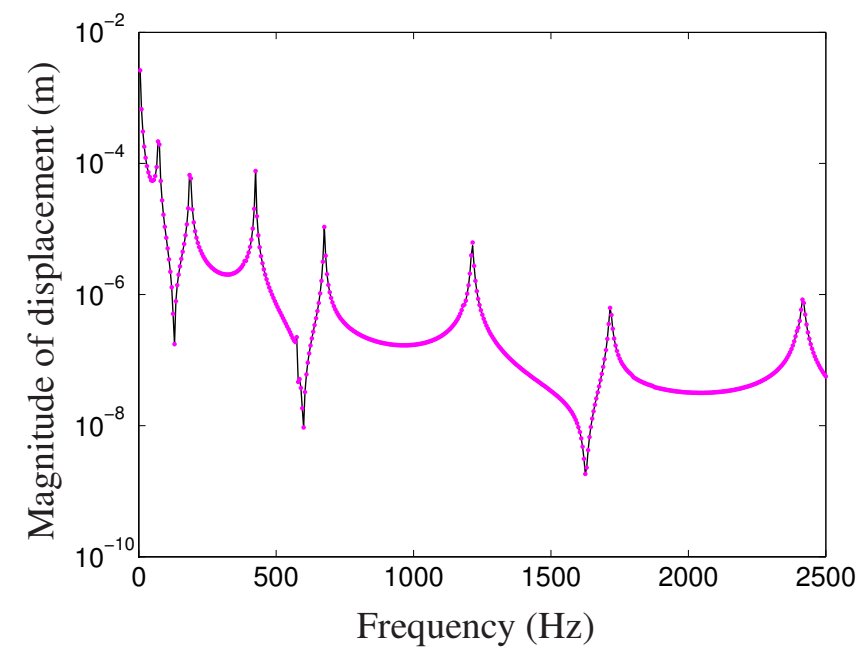

Figure 10: FRF of the assembly made up of a periodic structure with 36 substructures and a periodic structure with 60 substructures : (black solid line) FE method; (pink dots) proposed approach.

\subsubsection{Comparison with the theory of cyclic symmetry}

Again, the theory of cyclic symmetry may be considered. Its application to the study of coupled structures is not so straightforward however, and requires the assumption that the coupling effects between the so-called harmonic modes of the periodic structures are not taken into account (see [2, 22] for further details). The related FRF is shown in Figure 11 along with the FE solution. It is seen that the theory of cyclic symmetry suffers from a lack of accuracy above 
$1000 \mathrm{~Hz}$. In fact, the mean relative error is $12.6 \%$ which appears to be 30 times greater than the proposed approach. The computational cost of the theory of cyclic symmetry still remains interesting even compared to the proposed approach, i.e., 3600 s. However, the theory of cyclic symmetry is much less efficient than the proposed approach. As it turns out, the potentiality of the theory of cyclic symmetry to tackle more complex problems, i.e., assemblies made up of several periodic structures, is jeopardized, i.e., large errors are expected when calculating the dynamic response of such systems. This is not the case with the proposed approach which is a priori exact. Indeed, the full wave mode basis of each periodic structure, which is issued from the WFE method, is taken into account to build the matrix of dynamic flexibility modes. In other words, no assumption regarding (wave mode) basis reduction is made when implementing the proposed approach. The considered wave bases, for the two periodic structures, are complete in the sense that they exactly describe their displacement and force vectors.

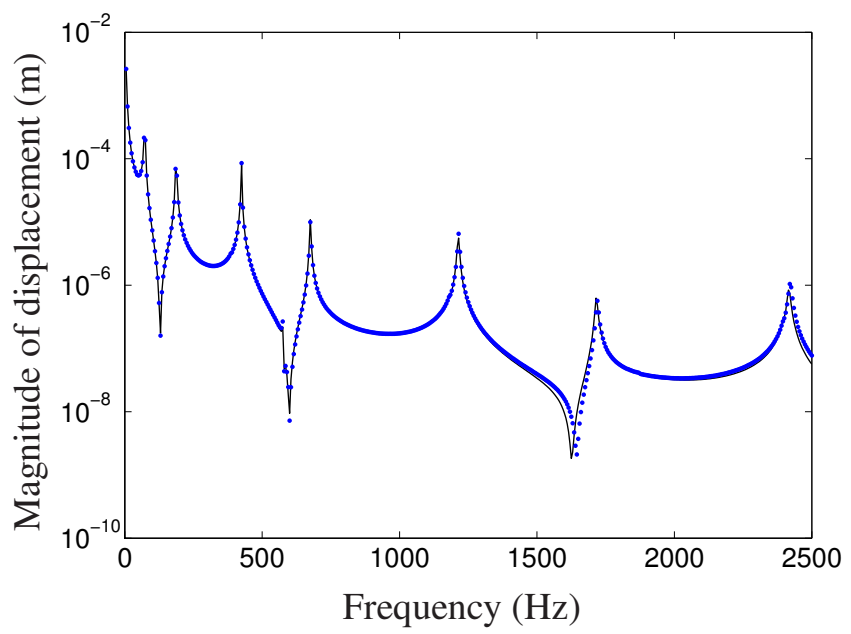

Figure 11: FRF of the assembly made up of a periodic structure with 36 substructures and a periodic structure with 60 substructures : (black solid line) FE method; (blue dots) theory of cyclic symmetry.

\section{CONCLUSIONS}

A wave-based numerical approach has been proposed for modeling periodic structures with cyclic symmetry, and assemblies made up of those structures. In the present framework, the WFE method has been considered to compute the wave modes of periodic structures with cyclic symmetry. The wave modes constitute an efficient means to build the matrices of dynamic flexibility modes of the structures. The modeling of assemblies made up of several periodic structures involves considering a matrix problem of small size whose resolution can be quickly achieved. Numerical experiments have been proposed which clearly highlight the potentiality of the proposed approach to handle complex systems made up of several periodic structures, but also, other structural components which are not necessarily periodic.

\section{REFERENCES}

[1] O. C. Zienkiewicz, R. L. Taylor, The Finite Element Method (first volume), ButterworthHeinemann, fifth edition, Oxford, 2000. 
[2] E. Balmes, I. Bucher, Accounting for rotation in a multi-stage cyclo-symmetric model a case study, Proceedings of the 24th International Conference on Noise and Vibration engineering (ISMA2010), Leuven, Belgium (2010) 1058-1064.

[3] J.-M. Mencik, M. N. Ichchou, A substructuring technique for finite element wave propagation in multi-layered systems, Computer Methods in Applied Mechanics and Engineering 197 (6-8) (2008) 505-523.

[4] B. Mace, D. Duhamel, M. Brennan, L. Hinke, Finite element prediction of wave motion in structural waveguides, Journal of the Acoustical Society of America 117 (2005) 2835.

[5] L. Gry, C. Gontier, Dynamic modelling of railway track: a periodic model based on a generalized beam formulation, Journal of Sound and Vibration 199 (4) (1997) 531-558.

[6] J. Signorelli, A. von Flotow, Wave propagation, power flow, and resonance in a truss beam, Journal of Sound and Vibration 126 (1) (1988) 127-144.

[7] Y. Waki, B. Mace, M. Brennan, Free and forced vibrations of a tyre using a wave/finite element approach, Journal of Sound and Vibration 323 (3-5) (2009) 737-756.

[8] E. Nobrega, F. Gautier, A. Pelat, J. D. Santos, Vibration band gaps for elastic metamaterial rods using wave finite element method, Mechanical Systems and Signal Processing 79 (2016) 192-202.

[9] J.-M. Mencik, M. N. Ichchou, Wave finite elements in guided elastodynamics with internal fluid, International Journal of Solids and Structures 44 (2007) 2148-2167.

[10] E. Manconi, B. Mace, R. Gaziera, Wave finite element analysis of fluid-filled pipes, Proceedings of NOVEM 2009 "Noise and Vibration: Emerging Methods", Oxford, UK.

[11] Q. Serra, M. N. Ichchou, J. F. Deü, Wave properties in poroelastic media using a Wave Finite Element Method, Journal of Sound and Vibration 335 (2015) 125-146.

[12] J.-M. Mencik, D. Duhamel, A wave-based model reduction technique for the description of the dynamic behavior of periodic structures involving arbitrary-shaped substructures and large-sized finite element models, Finite Elements in Analysis and Design 101 (2015) $1-14$.

[13] P. Silva, J.-M. Mencik, J. Arruda, Wave finite element-based superelements for forced response analysis of coupled systems via dynamic substructuring, International Journal for Numerical Methods in Engineering 107 (6) (2016) 453-476.

[14] J.-M. Mencik, On the low- and mid-frequency forced response of elastic systems using wave finite elements with one-dimensional propagation, Computers and Structures 88 (1112) (2010) 674-689.

[15] D. Duhamel, B. Mace, M. J. Brennan, Finite element analysis of the vibrations of waveguides and periodic structures, Journal of Sound and Vibration 294 (1-2) (2006) 205-220.

[16] Y. Waki, B. Mace, M. Brennan, Numerical issues concerning the wave and finite element method for free and forced vibrations of waveguides, Journal of Sound and Vibration 327 (1-2) (2009) 92-108. 
[17] D. Mead, The forced vibration of one-dimensional multi-coupled periodic structures: An application to finite element analysis, Journal of Sound and Vibration 319 (2009) 282-304.

[18] J.-M. Mencik, M. N. Ichchou, Multi-mode propagation and diffusion in structures through finite elements, European Journal of Mechanics - A/Solids 24 (5) (2005) 877-898.

[19] W. X. Zhong, F. W. Williams, On the direct solution of wave propagation for repetitive structures, Journal of Sound and Vibration 181 (3) (1995) 485-501.

[20] J.-M. Mencik, New advances in the forced response computation of periodic structures using the wave finite element (WFE) method, Computational Mechanics 54 (3) (2014) 789-801.

[21] M. Legrand, C. Pierre, P. Cartraud, J.-P. Lombard, Two-dimensional modeling of an aircraft engine structural bladed disk-casing modal interaction, Journal of Sound and Vibration 319 (1-2) (2009) 366-391.

[22] D. Laxalde, J.-P. Lombard, F. Thouverez, Dynamics of Multistage Bladed Disks Systems, Journal of Engineering for Gas Turbines and Power 129 (4) (2007) 1058-1064. 